\title{
The PAV trial: Does lactobacillus prevent post-antibiotic vulvovaginal candidiasis? Protocol of a randomised controlled trial [ISRCTN24 | 4 | 277]
}

\author{
Marie Pirotta*1, Jane Gunn ${ }^{1}$, Patty Chondros', Sonia Grover ${ }^{2}$, Susan Hurley ${ }^{3}$ \\ and Suzanne Garland 4
}

Address: ${ }^{1}$ Department of General Practice, University of Melbourne, 200 Berkeley Street, Carlton, Victoria, Australia, 3053, ${ }^{2}$ The Royal Women's Hospital, Women's \& Children's Health, 132 Grattan Street, Carlton, Victoria, Australia, 3053, ${ }^{3}$ Bainbridge Consultants, 532 Brunswick St, North Fitzroy, Victoria, Australia 3068 and ${ }^{4}$ Department of Microbiology and Infectious Diseases, The Royal Women's Hospital, Women's \& Children's Health, 132 Grattan Street, Carlton, Victoria, Australia, 3053

Email: Marie Pirotta* - mpirotta@unimelb.edu.au; Jane Gunn - j.gunn@unimelb.edu.au; Patty Chondros - p.chondros@unimelb.edu.au; Sonia Grover - sonia.grover@rch.org.au; Susan Hurley - susanhurley@bigpond.com; Suzanne Garland - suzanne.garland@wch.org.au

* Corresponding author

Published: 28 March 2004

BMC Family Practice 2004, 5:5

This article is available from: http://www.biomedcentral.com/I47/-2296/5/5

(c) 2004 Pirotta et al; licensee BioMed Central Ltd. This is an Open Access article: verbatim copying and redistribution of this article are permitted in all media for any purpose, provided this notice is preserved along with the article's original URL.

\begin{abstract}
Background: Complementary and alternative medicines are used by many consumers, and increasingly are being incorporated into the general practitioner's armamentarium. Despite widespread usage, the evidence base for most complementary therapies is weak or non-existent. Post-antibiotic vulvovaginitis is a common problem in general practice, for which complementary therapies are often used. A recent study in Melbourne, Australia, found that $40 \%$ of women with a past history of vulvovaginitis had used probiotic Lactobacillus species to prevent or treat postantibiotic vulvovaginitis. There is no evidence that this therapy is effective. This study aims to test whether oral or vaginal lactobacillus is effective in the prevention of post-antibiotic vulvovaginitis.

Methods/design: A randomised placebo-controlled blinded $2 \times 2$ factorial design is being used. General practitioners or pharmacists approach non-pregnant women, aged 18-50 years, who present with a non-genital infection requiring a short course of oral antibiotics, to participate in the study. Participants are randomised in a four group factorial design either to oral lactobacillus powder or placebo and either vaginal lactobacillus pessaries or placebo. These interventions are taken while on antibiotics and for four days afterwards or until symptoms of vaginitis develop. Women self collect a vaginal swab for culture of Candida species and complete a survey at baseline and again four days after completing their study medications. The sample size (a total of 496 - 124 in each factorial group) is calculated to identify a reduction of half in post-antibiotic vulvovaginitis from $23 \%$, while allowing for a $25 \%$ drop-out. An independent Data Monitoring Committee is supervising the trial. Analysis will be intention-to-treat, with two pre-specified main comparisons: (i) oral lactobacillus versus placebo and (ii) vaginal lactobacillus versus placebo.
\end{abstract}

\section{Background}

Use of complementary and alternative medicines (CAM) by the lay public and doctors remains a topic of contro- versy. In Australia and the United States, the estimated annual expenditure associated with CAM usage is \$AUD2.3 billion and \$US27.1 billion respectively, which 
is more than that spent on prescription medicines $[1,2]$. There is a relative lack of an evidence-base for CAM $[3,4]$, which was emphasised by recent calls from a Health Minister for an investigation into whether health insurance funds should reimburse for use of unproven CAM therapies [5]. However, scant resources are available for CAM research [6,7] and the accepted method of assessing medical therapies, randomised controlled trials, can be challenging to apply to CAM $[7,8]$.

A common condition of concern to women, the largest consumers of CAM $[1,9]$, is post-antibiotic vulvovaginitis (PAV) [10], usually caused by Candida albicans [11]. In our recent survey of Australian women, $40 \%$ of a sample of 751 with a past history of vulvovaginitis had used yoghurt or lactobacillus orally and/or vaginally to prevent PAV [10]. Other countries have found similar high rates of use for vaginal symptoms of lactobacillus products $[12,13]$, which are also recommended by natural medicine practitioners for vulvovaginitis [14].

Although lactobacillus is commonly recommended and used for PAV, a thorough literature search using the terms lactobacillus, vagina, Candida and Cochrane Collaboration search strategies for randomised controlled trials, revealed no trials which had tested whether lactobacillus is effective in the prevention of PAV, although several trials have examined lactobacillus in recurrent vulvovaginitis. Most of these trials were methodologically poor (inadequate allocation concealment, not randomised, no controls and/or not intention-to-treat analysis) [15-18]; in the most recent trial in women with HIV, the lactobacillus arm failed to reach statistical significance in the prevention of vulvovaginal candidiasis [19].

This study aims to test in a randomised controlled trial whether oral and/or vaginal lactobacillus preparations can prevent post-antibiotic vulvovaginitis.

\section{Methods/design \\ Study design}

The PAV study uses a randomised controlled blinded $2 \times$ 2 factorial design, with four intervention groups (see figure 1). The factors are oral lactobacillus, oral placebo, vaginal lactobacillus and vaginal placebo. The design and analysis assumes that there will be no interaction between the oral and vaginal interventions. There are two pre-specified main comparisons: (i) oral lactobacillus versus placebo and (ii) vaginal lactobacillus versus placebo.

\section{Study population}

Inclusion criteria

The inclusion criteria are: women aged between 18 and 50 years of age [20,21]; who are experiencing a non-genital infection; requiring a short-course of oral antibiotics or have commenced this within the 48 hours preceding enrolment; and English-speaking - able to speak, read and write in English sufficiently to provide informed consent and complete surveys.

\section{Exclusion criteria}

Exclusion criteria are: pregnancy; experiencing any vaginal symptoms at recruitment; unwilling or unable to provide two self-collected low vaginal swabs (at recruitment and on completion of trial); using or have used vaginal antifungal treatments in the past two weeks [21]; have taken antibiotics in the past month [22]; unwilling to stop taking other lactobacillus products during the trial; or immunocompromised, as there are rare reports in the literature of lactobacilli causing endocarditis and bacteraemia [23].

\section{Sample size}

The sample size calculation is based on a local incidence rate of PAV of $23 \%$ [ 10 ] with a power of $80 \%$ and significance level of $5 \%$ for a 2 -sided test. To detect a clinically meaningful $50 \%$ reduction in the incidence of PAV, a total of 93 women are required in each arm of the study. Allowing for a $25 \%$ drop out rate, 124 women are required in each of the four arms, which equates to a planned total of 496 women.

\section{Randomisation}

\section{Sequence}

Block randomisation in batches of eight using computergenerated random numbers is being used to assign women to one of the four combinations of trial intervention. Block randomisation was chosen to ensure that the experimental groups would not become unbalanced if the rate of recruitment at sites differed greatly. A total of eight in each batch is used as most general practice clinics have limited space in their vaccination refrigerators and eight intervention pairs is considered the most that could be reasonably accommodated at one time.

\section{Allocation concealment}

A research fellow with no other role in the project is overseeing the labelling and packing of all the trial medications and holding the randomisation schedule until the code is broken.

\section{Implementation}

Batches of eight intervention pairs, numbered sequentially, are provided to community-based recruiters or kept centrally in a dedicated refrigerator at the Department of General Practice for use by the mobile recruitment team. Recruiters are instructed to use the lowest numbered intervention first and then continue consecutively. 


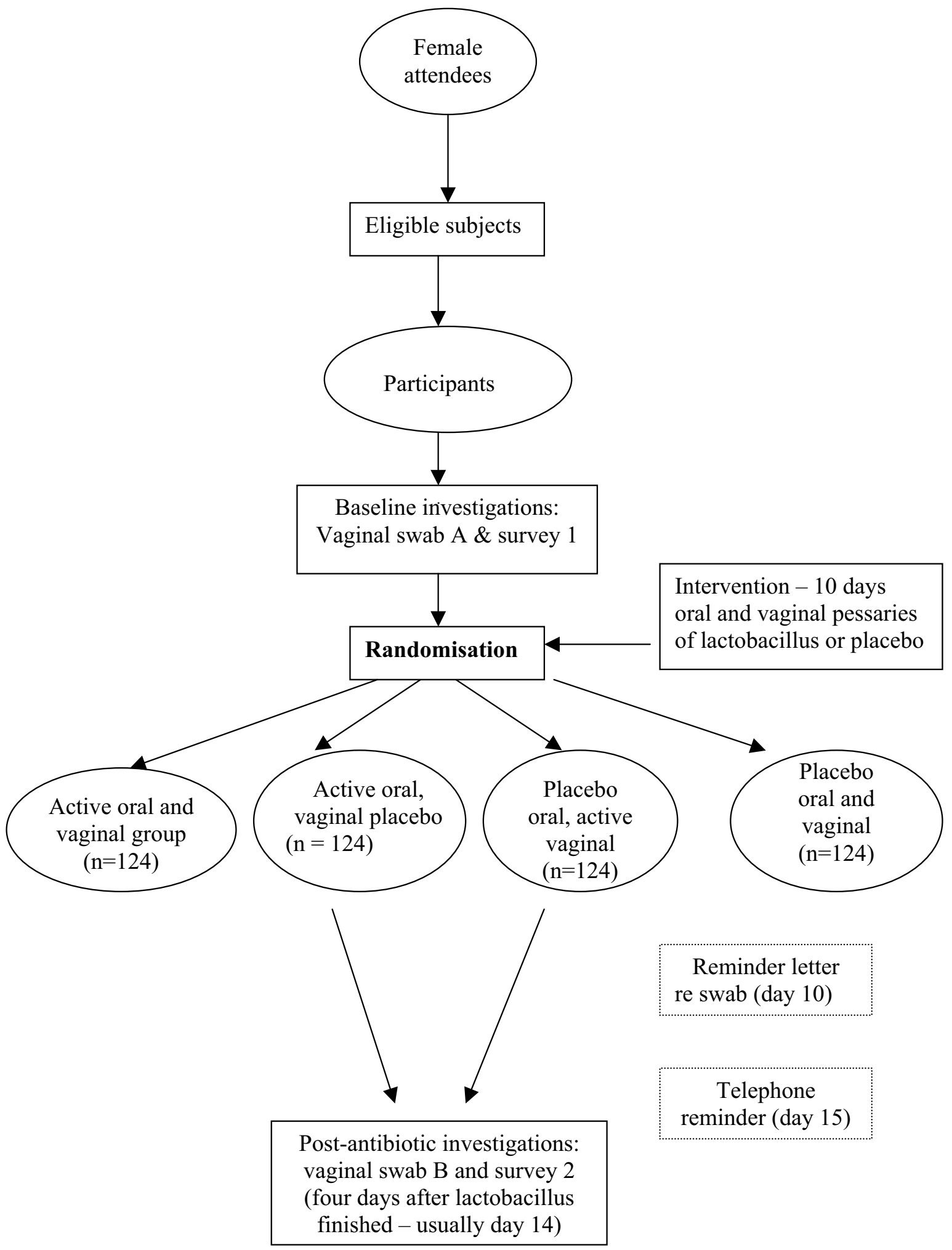

Figure I

Trial design 


\section{Blinding}

Participants, recruiters and microbiology staff responsible for processing the participants' specimens are blinded to group allocation. The success of masking of participants is checked with a question in the final survey. Community recruiters do not have access to microbiology results and microbiologists do not know the symptom status or identity of participants.

\section{Interventions}

Many women report using yoghurt to prevent or treat post-antibiotic vulvovaginitis. However, this was not seriously considered as an option for this study, due to its instability [24], relatively short shelf life, lower standard of quality control (manufacture is regulated by food rather than pharmaceutical standards [16]); presumed lack of acceptability to some women for vaginal use; and difficulty of standardising a per vaginum dose.

The interventions being tested in the $2 \times 2$ factorial design are:

(i) oral powder: either Lactobac - containing Lactobacillus rhamnosus and Bifidobacterium longum or placebo - maltodextrin powder; and

(ii) vaginal pessaries: either Femilac - L. rhamnosus, L. delbrueckii, L. acidophilus and Streptococcus thermophilus or placebo - malto-dextrin pessaries.

The oral dose chosen is half a teaspoon of powder twice daily 20 minutes before meals; the per vaginum regimen is one pessary to be inserted high into the vagina with a supplied applicator at bedtime for ten days, whilst taking, and continued for four days after completion of, the antibiotic course. Ten days is felt to be a reasonable balance between a minimum to be effective and a maximum, to which women would adhere for prevention of a possible side-effect.

Women enrolled in the trial sometimes require additional days of antibiotics for various clinical reasons. When this occurs, the courses of oral and vaginal interventions are extended to last until four days after the antibiotics cease, if the participant agrees. To deal with this circumstance, the research fellow responsible for maintaining allocation concealment directs which intervention code numbers to use to provide the woman with additional trial medication, which preserves the same study group to which the woman was originally allocated.

\section{Recruitment}

\section{Recruitment network}

Recruitment for the trial is through a network of community-based general practice clinics and pharmacies. Gen- eral practitioners (GPs) have been trained to identify potential participants and to introduce the trial to them. If women are eligible and consent to participating, then the GP could either recruit themselves, or refer to a mobile recruitment team, consisting of MP, POM and KN. Some community-based pharmacists are also trained to inform women about the trial, when dispensing antibiotics.

\section{Enrolment of participants}

Recruiting GPs identify eligible women in their clinical practice and invite them to consider participation in the trial, after provision of sufficient information to make an informed decision (see appendix 1 (Additional file 1) for the plain language statement). Women who meet the eligibility criteria and agree to participate are required to give written informed consent (see appendix 2 (Additional file 2) for the consent form). All women eligible to participate, including women who decline to participate, are asked for permission for the GP to complete the PAV Recruitment sheet, that is a log of all potential participants to check whether the study population is representative of the entire eligible population in key criteria related to the outcomes of interest. Recruiting GPs also obtain demographic and relevant past and current medical data, particularly data relevant to risk factors for developing vulvovaginal candidiasis (see appendix 3 (Additional file 3) Recruiter Survey).

To refer the woman to the mobile recruitment team, the GP or pharmacist can provide women with a 'spotter flyer' (see appendix 4 (Additional file 4) spotter flyer) with information about the trial and contact details. The GP, pharmacist or woman herself can call the mobile recruitment team on a mobile telephone dedicated to the trial. The team member will talk to the woman, establish her eligibility, explain the trial and then arrange a mutually agreeable time to recruit at the woman's home or workplace on that day, in a similar manner to that described for general practitioners. The mobile recruitment team is available seven days a week, as it is essential to commence the interventions as soon as possible after the course of antibiotics has commenced.

\section{Data collection}

At enrolment, participants complete a baseline survey (see appendix 5 (Additional file 5) survey 1) and, after appropriate written instruction (see appendix 6 (Additional file 6)'Instructions for taking your vaginal swab'), provide their own vaginal swab ("swab A"). A second swab ("swab B") and survey (see appendix 7 (Additional file 7) survey 2) are required four days after completing the intervention, generally 14 days after recruitment or at the time of developing symptoms of vaginitis. 
After randomisation into a study group, women are supplied with a unique PAV identity number, a PAV Take Home Kit and their intervention powder and pessaries. The PAV Take Home Kits, all their contents and the intervention medications each have the same PAV identity number. The kits contain surveys 1 and 2 with reply paid envelopes, swabs A and B with labelled microbiology request forms and specimen bags, the laboratory's address and courier instructions printed on courier envelopes, a prepaid courier voucher, a Frequently Asked Questions brochure (see appendix 8 (Additional file 8) FAQs), a PAV logo sticker with the date when swab $\mathrm{B}$ is due written by the recruiter and two PAV logo refrigerator magnets with PAV team contact details.

The baseline survey requests basic demographic information such as age, country of birth, level of education and postcode; self-assessment of likelihood of PAV; type of antibiotic prescribed and infection; other risk factors for developing vulvovaginal candidiasis; weight and height to calculate body mass index (BMI); whether sexually active and any other medical conditions. Survey 2 requests information about participants' adherence to the antibiotic, oral and vaginal intervention regimes; any symptoms of PAV or side-effects of the intervention; whether participants think they were allocated real or placebo interventions and how effective the interventions were.

The microbiology specimens are collected and transported within 24 hours to the Royal Children's Hospital laboratory, Women's and Children's Health, Melbourne, Australia. Swabs at recruitment are either transported by the mobile team or by local pathology companies. A courier company collects swab Bs from a location convenient to the participants.

Self-collected vaginal swabs are initially rolled on to a glass slide for Gram stain examination microscopically. The swabs are then rolled on to a half CHROMagar medium (CHROMagar, Paris) [25] and incubated at $35^{\circ} \mathrm{C}$. for two days. Candida albicans, C. glabrata, and C. krusei are identified by specific colour of colonies on CHROMagar; other candida species are identified by ID32C kit (BioMérieux, France) [26].

\section{Follow-up of participants}

There are several phases of follow-up once women enter the trial.

- Women recruited by GPs are telephoned the day after recruitment to check that they are clear about their commitment, still consent to participate and to answer any queries.
- A letter is mailed to all women approximately one week after recruitment, to welcome them to the study and reconfirm the due date for swab $B$.

- Participants who do not return survey 2 or swab B are followed-up within one week with telephone calls, and if necessary letters, to obtain information about any symptoms of PAV.

- All women who report no symptoms of PAV at day 14, but whose swab B culture Candida species, are contacted at least one week after trial completion to ascertain whether symptoms of PAV developed.

Women who develop symptoms of PAV during the trial Women have printed instructions in their PAV Kit about how to respond if symptoms of vaginitis develop during the trial. The main message is to obtain swab $\mathrm{B}$ prior to any treatment for symptoms. Women may contact a member of the research team on the mobile PAV telephone number, who will be available daily until $10 \mathrm{pm}$ and can arrange for either a courier or herself to collect the swab that day for delivery to the laboratory. Once the swab is taken, even prior to discussing the situation with the PAV team, women are encouraged to treat themselves in their usual manner. Women may elect to attend their GP to have swab B taken.

\section{Quality control}

\section{Run-in phase}

The initial 25 participants and their recruiting general practitioners have been interviewed by telephone by MP to seek ways to improve the study method.

\section{Intervention}

Adequate storage of the trial medications requires refrigeration between 2 and 8 degrees Celsius. Recruiting clinics are required to ensure adequate storage of their eight pairs of PAV medication in the clinic vaccine refrigerator, which have continual temperature recordings to comply with practice accreditation standards.

Prior to study commencement of the trial, once during recruitment and again at the end of the trial, randomly chosen intervention medications are subjected to independent microbiological examination (Royal Children's Hospital, Melbourne, microbiology laboratory), to confirm that the lactobacillus species are viable and that the labelling of active and placebo medication is correct.

\section{Microbiology}

All microbiological specimens are being processed in the one laboratory (Department of Microbiology and Infectious Diseases, Royal Children's Hospital, Women's and Children's Health, Melbourne, Australia) to ensure con- 
sistency of processing and reporting. Even if women develop PAV symptoms and opt to attend their GP for management, the GPs are requested to forward the specimens to the preferred trial laboratory.

\section{Data entry}

The trial databases are constructed with stringent checking criteria to prevent mistakes in data entry. Research assistants receive adequate training and support to ensure thorough understanding of the process for data entry. Ten percent of surveys are audited to ensure high standards of accuracy and reliability of interpretation prior to any data processing. Data cleaning and checking is undertaken using several strategies, commencing with the construction of the database in Microsoft Access and also using Stata [27].

\section{Women's participation}

Several questions in survey 2 check the process of women's participation in the trial. These questions regard compliance with and side-effects of both antibiotics and study medications; the experience of providing a vaginal swab; willingness to pay for the study medications if they are effective; and participants' opinions as to whether they are allocated to lactobacillus or placebo medications and whether the medications were effective.

\section{Data monitoring committee}

An independent Data Monitoring Committee oversees the conduct of the trial. The committee comprises of Professor Judith Lumley (chair - Director of the Centre for Study of Mothers' and Children's Health, La Trobe University and professor of perinatal epidemiology), Dr Susan Hurley (pharmacologist and epidemiologist), Dr Sonia Grover (gynaecologist) and Ms Patty Chondros (non-voting member and statistician for the trial). This committee will meet three times, with two interim analyses review meetings scheduled to occur when 126 and 252 women have been recruited. O'Brien and Fleming's procedure and stopping rules [28] will be employed with the nominal p value of 0.0005 at the first meeting and 0.014 at the second. To allow for the loss of power due to two interim analyses and the final analysis, the target sample of women providing outcome data is inflated by a factor of 1.017 from 372 to 380 women. The Data Monitoring Committee will examine reports of side-effects of the interventions at each of its meetings.

\section{Outcomes}

The primary outcome is symptomatic vulvovaginal candidiasis, which is defined as participants' report of symptoms (that is answer 'yes' to a question about symptoms of 'thrush' in survey 2: vaginal itch, irritation with or without a discharge) and swab $\mathrm{B}$ culture positive for Candida species.

\section{Data analysis}

Analysis is intention-to-treat with two pre-specified main comparisons: (i) oral lactobacillus versus placebo and (ii) vaginal lactobacillus versus placebo, using Stata version 7 [27]. Categorical baseline characteristics will be described as frequencies and percentages for each factorial group, to assess whether potential confounders are balanced across the groups. Differences in baseline characteristics between women who provided outcome data and those who did not will be examined using Pearson chi-square statistic for categorical variables and two-sample t-test for age. The numbers of PAV cases will be reported as percentages with $95 \%$ confidence intervals (CIs). Logistic regression will be used to examine whether active pessaries or active powder are effective in preventing PAV. The results of the outcome will be reported as odds ratios (ORs) with 95\% CIs. Treatment interaction between oral and vaginal interventions will also examined in the logistic model.

Sensitivity analyses will be carried out whereby participants with incomplete outcome data (no swab B) who report symptoms of PAV or who provide no outcome data are treated as non-PAV cases in the first scenario and as PAV cases in the second scenario. Contacted non-completers who report no symptoms of PAV will be treated as non-PAV cases in both scenarios.

\section{Trial organisation}

Ethics and ethical issues

The trial has received ethics approval from both the Royal Australian College of General Practitioners and the Royal Women's Hospital, Melbourne, Australia.

Women are required to provide written informed consent (Additional file: appendix 2) prior to entering the trial and are free to withdraw at any time.

As both interventions being tested in the trial are available over-the-counter, it is not anticipated that there will be any major risks to participants. Women may benefit if randomised to an active intervention, but this is not known. The inconveniences to participants include providing two vaginal swabs and taking oral and vaginal medications for ten days. Side-effects of the interventions will be monitored and reviewed at each of the Data Monitoring Committee's meetings. Any serious problems will be reported to both of the ethics committees involved.

\section{Personel}

The trial investigators are Dr Marie Pirotta (Department of General Practice, University of Melbourne), Associate Professor Jane Gunn (Department of General Practice, University of Melbourne), Associate Professor Suzanne Garland (Department of Microbiology \& Infectious Diseases, Royal Women's Hospital, Melbourne), Dr Susan 
Hurley, Dr Sonia Grover (Senior Obstetrician, Royal Women's Hospital, Melbourne), Ms Patty Chondros (statistician, Department of General Practice, University of Melbourne) and Ms Cate Nagle holds the randomisation schedule. The research assistants are Paula O'Malley, Kitty Novy and Belinda Clarke.

The reference group comprises Dr Lisa Amir, general practitioner and lactation consultant with a research interest in candidiasis of the nipple; Dr Trish Boetto, general practitioner and practitioner of Nutritional and Environmental medicine; Dr Liz Gorczyca, scientist at RMIT with a research interest in food-based lactobacilli; Ms Gail Roberts, a consumer, who has worked in various health related areas and been a board member of the Health Issues Centre and Mrs Keri Jones, Assistant Lecturer, Department of Pharmaceutics, Victorian College of Pharmacy, Parkville.

\section{Administration}

A database of recruiters is constructed and maintained to keep track of all the recruiting general practitioners and pharmacists. A research assistant is responsible for providing feedback to recruiters after each referral. A successful referral receives a telephone call and then a postcard advising the recruiter of the success of the referral. Unsuccessful referrals receive a postcard advising why the referral failed in order to remind referrers if trial criteria have not been fulfilled. MP and POM create and mail regular newsletters to the recruiting network to maintain the network of recruiters' enthusiasm and support for the trial.

When a new participant enters the trial, it is necessary to ensure all the initial paperwork is complete (consent form, survey 1, swab A and recruiter survey), arrange for collection of trial data as soon as possible from the clinic to ensure there are no problems, and that swab A has arrived at the laboratory. The survey 1 , recruiters survey and consent form are then checked, coded, entered into the Access database and filed in a locked filing cabinet. Participants receive a welcome call within 24 hours if enrolled by a community-based recruiter.

\section{Trial registration}

The importance of trial registration has been emphasized in the literature as a method to reduce publication bias [29]. The PAV trial was registered in the UK (registration number number: ISRCTN24141277).

\section{Competing interests}

Nutrition Care (Melbourne) and Institut Rosell (Montreal) provided the medications and placebos being used in the trial and also partially funded the trial. However, they have no access to data collected from the trial or input into the study design, analysis or reporting of the results.

\section{Authors' contributions}

Study conception: MP, JG

Study design: MP, JG, PC, SGarland, SGrover, SH

Initial grant application: MP, JG, SGarland, SGrover, SH

Initial protocol and questionnaire drafts: MP

Questionnaire development, piloting and completion: MP, JG.

Coordination of the trial: MP

Ongoing review of trial processes: MP, JG

Development of data collection processes: MP, PC

Data collection, management and analysis: MP, PC

Supervision of microbiological processing of clinical samples: SGarland

Draft of trial protocol manuscript: MP

All authors have read and approved the final manuscript.

\section{Additional material}

\section{Additional File 1}

Appendix 1: plain language statement

Click here for file

[http://www.biomedcentral.com/content/supplementary/14712296-5-5-S1.pdf]

\section{Additional File 2}

Appendix 2: consent form

Click here for file

[http://www.biomedcentral.com/content/supplementary/14712296-5-5-S2.pdf]

\section{Additional File 3}

Appendix 3: recruiter survey

Click here for file

[http://www.biomedcentral.com/content/supplementary/14712296-5-5-S3.pdf]

\section{Additional File 4}

Appendix 4: spotter flyer

Click here for file

[http://www.biomedcentral.com/content/supplementary/14712296-5-5-S4.doc]

\section{Additional File 5}


Appendix 5: survey 1

Click here for file

[http://www.biomedcentral.com/content/supplementary/1471-

2296-5-5-S5.doc]

\section{Additional File 6}

Appendix 6: instructions to take vaginal swab

Click here for file

[http://www.biomedcentral.com/content/supplementary/1471-

2296-5-5-S6.doc]

\section{Additional File 7}

Appendix 7: survey 2

Click here for file

[http://www.biomedcentral.com/content/supplementary/1471-

2296-5-5-S7.doc]

\section{Additional File 8}

Appendix 8: frequently asked questions

Click here for file

[http://www.biomedcentral.com/content/supplementary/14712296-5-5-S8.doc]

\section{Acknowledgements}

We thank all the women and general practitioners who assisted in piloting the study materials in this study and are taking part in the trial. Nutrition Care and Institut Rosell kindly donated the medications and placebos. Mayne Health and Gribbles Pathology provided free transportation of some of the microbiological specimens. Thanks to Prof Judith Lumley for being the chair of the Data Monitoring Committee and Ms Cate Nagle for managing the randomisation schedule.

This project is funded by a Quality Use of Medicine Scholarship funded by the Australian Commonwealth Department of Health and Aging; the Shepherd Foundation; the Royal Australian College of General Practitioners; Nutrition Care; Institut Rosell; Health Care Network; and Mr Sean Howard.

\section{References}

I. MacLennan A, Wilson D, Taylor A: The Escalating Cost and Prevalence of Alternative Medicine. Preventive Medicine 2002, 35:166-173.

2. Eisenberg D, Davis R, Ettner S, Appel S, Wilkey S, Van Rompay M Kessler R: Trends in alternative medicine use in the United States, 1990-1997. JAMA 1998, 280:1569-1575.

3. Anonymous: Complementary medicine: time for critical engagement. Lancet 2000, 356:2023.

4. Angell M, P Jerome: Alternative Medicine - The Risks of Untested and Unregulated Remedies. New England Journal of Medicine 1998, 339:839-84I.

5. Wroe D: Alternative health care under fire. The Age Melbourne; 2003:5.

6. McCarthy M: US panel calls for more support of alternative medicine. Lancet 2002, 359:1213.

7. Nahin R, Straus S: Research into complementary and alternative medicine: problems and potential. BMJ 200I, 322:16I-I64.

8. Ernst $\mathrm{E}$ : Obstacles to research in complementary and alternative medicine. MJA 2003, 179:279.

9. Adams J, Sibbritt D, Easthope G, Young A: The profile of women who consult alternative health practitioners in Australia. MJA 2003, I 79:297-300.

10. Pirotta M, Gunn J, Chondros P: "Not thrush again!" Women's experience of post-antibiotic vulvovaginitis. Med J Aust 2003, 1 79:43-46.
II. Bluestein D, Rutledge C, Lumsden L: Predicting the occurrence of antibiotic-induced candidal vaginitis (AICV). Fam Pract Res J | 99|, I I:319-326.

12. Nyirjesy P, Weitz M, Grody T, Lorber B: Over-the-counter and alternative medicines in the treatment of chronic vaginal symptoms. Obstet Gynecol 1997, 90:50-53.

13. Lipsky MS, Taylor C: The use of over-the-counter antifungal vaginitis preparations by college students. Family Medicine 1996, 28:493-495.

14. Pizzorno L, Pizzorni J, Murray M: Natural Medicine Instructions for Patients. Churchill Livingstone; 2002.

15. Friedlander A, Druker MM, Schachter A: Lactobacillus acidophillus and vitamin $B$ complex in the treatment of vaginal infection. Panminerva Medica 1986, 28:5I-53.

16. Hilton E, Isenberg HD, Alperstein P, France K, Borenstein MT: Ingestion of yogurt containing Lactobacillus acidophilus as prophylaxis for candidal vaginitis. Annals of Internal Medicine 1992, I 16:353-357.

17. Hilton E, Rindos P, Isenberg HD: Lactobacillus GG vaginal suppositories and vaginitis. J Clin Microbiol I995, 33: I433.

18. Shalev E, Battino S, Weiner E, Colodner R, Keness Y: Ingestion of yogurt containing Lactobacillus acidophilus compared with pasteurized yogurt as prophylaxis for recurrent candidal vaginitis and bacterial vaginosis. Arch Fam Med 1996, 5:593-596.

19. Williams AB, Yu C, Tashima K, Burgess J, Danvers K: Evaluation of two self-care treatments for prevention of vaginal candidiasis in women with HIV. Journal of the Association of Nurses in AIDS Care 200I, I 2:5I-57.

20. Sobel JD, Faro S, Force RW, Foxman B, Ledger WJ, Nyirjesy PR, Reed $B D$, Summers PR: Vulvovaginal candidiasis: epidemiologic, diagnostic, and therapeutic considerations. Am J Obstet Gynecol 1998, I78:203-2II.

2I. Dennerstein GJ, Ellis DH: Oestrogen, glycogen and vaginal candidiasis. Australian \& New Zealand Journal of Obstetrics \& Gynaecology. 200I, 4I:326-328.

22. MacDonald TM, Beardon PH, McGilchrist MM, Duncan ID, McKendrick $A D, M c D e v i t t D G$ : The risks of symptomatic vaginal candidiasis after oral antibiotic therapy. Quarterly Journal of Medicine 1993, 86:419-424.

23. McGroarty JA: Probiotic use of lactobacilli in the human female urogenital tract. FEMS Immunology \& Medical Microbiology 1993, 6:25I-264.

24. Gilliland M: Instability of Lactobacillus acidophilus in yogurt. J Diary Science 1977, 60:1394-1398.

25. Bernal S, Mazuelos EM, Garcia M, Aller Al, Martinez MA, MJ. Gutierrez: Evaluation of CHROMagar Candida medium for the isolation of presumptive identification of species of Candida of clinical importance. Diagn Microbiol Infect Dis 1996, 24:20I-204.

26. Hospenthal DR, Murray CK, Beckius ML, Green JA, Dooley DP: Persistence of pigment production by yeast isolates grown on CHROMagar Candida medium. I Clin Micro 2002, 40:4768-4770.

27. StataCorp: Stata Statistical Software. 7th edition. College Station, Texas, Stata Corporation; 200I.

28. O'Brien P, Fleming T: A multiple testing procedure for clinical trials. Biometrics 1979, 35:549-556.

29. Hetherington J, Dickersin K, Chalmers I, Meinert C: Retrospective and prospective identification of unpublished controlled trials: lessons from a survey of obstetricians and pediatricians. Pediatrics 1989, 84:374-380.

\section{Pre-publication history}

The pre-publication history for this paper can be accessed here:

http://www.biomedcentral.com/1471-2296/5/5/prepub 